\title{
N.I.P. \\ (NOUVEAUX INSTRUMENTS PROMOTIONNELS) ET PROMOTIONS DES MARQUES : VERS UNE SYNERGIE NÉCESSAIRE
}

\section{Franck Debos*}

Résumé : en terme de communication persuasive, les responsables Marketing ont jusqu'à présent eu tendance à opposer les techniques promotionnelles aux outils de fidélisation de la clientèle. Or, la mise en place des NIP (Carte cagnotte, Lot virtuel, Offre fédérative...) développés par les enseignes de distribution en collaboration (plus ou moins forcée il est vrai) avec les fabricants ainsi que d'autres techniques promotionnelles spécifiques aux marques comme la promotion "Quasi Publicitaire ", sont à la fois des outils d'accroissement des ventes à court terme mais aussi de fidélisation et de valorisation efficace du capital de marque des fabricants associés aux distributeurs.

Les NIP doivent alors être réellement intégré dans une véritable réflexion et stratégie Marketing des fabricants et être associées à leurs propres actions promotionnelles.

Mots-clés : promotion, fidélisation, capital Marque, partenariat Fabricants Distributeurs.
Sumarry: until recently, in the realm of persuasive communication marketing managers have tended to separate the notion of promotional techniques from that of tools aimed at creating customer loyalty.

However, the use of NPTs (lottery card, virtual tomobola, ....) developed by the big names in distribution in collaboration (more or less forced) with manufacturers, as well as the use of other promotional techniques specific to brands such as "quasi-advertising" promotions is not only a means of increasing sales in the short term and but also of creating customer loyalty and effectively raising the value of the brand capital of the manufacturers associated with the distributors.

The use of NPT must therefore be well thought-out within the framework of the marketing strategy of the manufacturers and NPTs must be applied alongside their own promotions.

Key words: promotional techniques, customer loyalty, brand capital, synergy Manufacturers Distributors .

* Professeur associé à l'I.U.T. de Nice, Site de Cannes. Chercheur au LAMIC. 
N.I.P. (Nouveaux instruments promotionnels) et promotions des marques : vers une synergie nécessaire

\section{Introduction}

En terme de communication persuasive, les responsables Marketing ont jusqu'à présent eu tendance à opposer les techniques promotionnelles aux outils de fidélisation de la clientèle.

La promotion, notamment à travers les VIP ou "vieux instruments promotionnels " regroupe les techniques de "Hard Selling " proposées par les marques (Lots, Réductions, Gratuité...) et aspire à développer les ventes à court terme en poussant le produit vers le consommateur à l'aide d'avantages directs ou différés. Par ses aspects réactifs et tactiques, cette forme de communication persuasive tend à créer chez le client un comportement de "Chasseur de primes".

A contrario les outils de fidélisation développés par les marques au travers des techniques liées au Marketing Interactif " one to one " (ou à tout le moins " one to few ") veulent développer une relation de confiance chez le consommateur qui se sentira comme un véritable " partenaire " de l'entreprise.

Ces actions de fidélisation permettent de valoriser le capital de marque que l'on peut définir comme "l'ensemble des associations de la marque fortes et transmissibles et leur capacité à influencer le comportement " (Leutheusser-1988)1 ${ }^{1}$. Ces outils sont associés à une véritable réflexion stratégique sur le devenir à moyen et long terme des marques considérées.

Or, la mise en place des NIP développés par les enseignes de distribution dans un premier temps en collaboration (plus ou moins forcée il est vrai) avec les fabricants ainsi que d'autres techniques promotionnelles spécifiques aux marques comme la promotion "Quasi Publicitaire ", sont aussi des outils de fidélisation et de valorisation efficace du capital de marque des fabricants associés aux distributeurs.

Nous présenterons dans une première partie les techniques de communication persuasive utilisées dans le but de fidéliser la clientèle, de valoriser le capital marque de l'entreprise, et centrée sur la notion d'interactivité 2 (DEBOS 2004).

Nous pourrons ainsi déterminer une première catégorie d'action promotionnelle découlant de cette communication interactive. Il s'agit des actions promotionnelles qui peuvent constituer une première tentative de conciliation entre les notions de promotion et de fidélisation au niveau de la stratégie Marketing de l'entreprise.

Dans un second temps nous aborderons les NIP (Nouveaux Instruments Promotionnels) développés par les distributeurs ainsi que les promotions à contenu d'image mise en place de façon concomitante par les marques fabricants. Nous tenterons de montrer de quelles façons ces outils de communication persuasive, complétés par les actions promo-relationnelles peuvent à la fois développer le Chiffre d'Affaires de l'entreprise à court-terme, mais aussi de fidéliser le consommateur à plus long terme et de consolider le capital marque de l'entreprise.

1. L. LEUTHEUSSER - 1988 - "Defining, Reassuring Managing Brand Equity": Working Paper pp88-104 CambridgeMarketing Science Institute.

2. F. DEBOS Sept 2004 - "L'impact de l'interactivité sur la communication commerciale de l'entreprise : vers l'animation de clans d'audience". 14e Conférence Internationale du RESER - Volume I - 580 pages. 
Il s'agira alors d'intégrer les NIP dans une véritable réflexion et stratégie Marketing des Marques fabricants.

\section{Les techniques de communication persuasive " fidélisantes "}

\subsection{L'importance de l'interactivité au niveau de la fidélisation des clients}

La communication interactive implique la mise en place d'une relation personnalisée et directe avec le client modifiant tous les process concernés par la vente des produits. Le recueil des informations et la gestion des mégabases de données permet de répondre aux aspirations individualisées du consommateur, de mettre en œuvre une production "sur mesure ", d'assurer la distribution/vente directe des produits et services, et de créer une véritable relation de communication personnalisée avec le consommateur.

C'est une nouvelle pratique des métiers de marketing qui se met en place, où l'échange est permanent entre le client et l'entreprise. L'effort doit porter sur la sélectivité des cibles, la fidélisation, l'individualisation nominale de l'offre vers le client et la capacité à capturer et à captiver un consommateur insaisissable. La communication doit créer l'intérêt et le trafic vers les offres des marques dans un monde où le consommateur ne se voit plus imposer la publicité et peut s'éclipser vers une autre offre aux moindres sentiments de lassitude.

Cette consommation s'intègre dans une stratégie de Marketing " one to one " dont nous pouvons rappeler les principes de base.

À ce niveau Don Peppers et Martha Rogers identifient cinq idées-forces pour définir cette tendance marketing 3 :

- L'ATTRACTION : il s'agit d'établir avec ses clients des liens interactifs permettant de mieux connaître et satisfaire leurs besoins et désirs individuels. De ce fait elle peut exercer sur ses clients une forte attraction, gage de fidélité et de croissance.

- LA DIFFÉRENCIATION : l'entreprise va distinguer ses clients à partir de deux critères essentiels; les besoins et désirs qu'ils expriment et la valeur économique qu'ils représentent. Elle pourra ainsi identifier les motivations d'achat des consommateurs et le profit potentiel de chaque client.

\section{- LA RELATION D'APPRENTIS-} SAGE : il faut tisser une relation privilégiée entre l'entreprise et son client. Celui-ci va renseigner l'entreprise sur son mode de vie, ses attentes et habitudes de consommation afin d'obtenir une réponse personnalisée.

\section{- LA PERSONNALISATION DE} MASSE : cette expression paradoxale recouvre la production en masse de produits aussi personnalisée que possible. Le plus souvent, elle consiste à assembler des éléments de base en fonction des commandes spécifiques au dernier moment.

- LA VALEUR STRATÉgIQUE DU CLIENT : cette notion recouvre le

3. D. PEPPERS - M. ROGERS 1999 " Le one to one : valorisez votre capital client ". Édition Organisation. 
N.I.P. (Nouveaux instruments promotionnels)

et promotions des marques : vers une synergie nécessaire

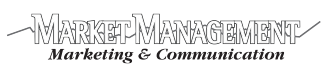

Chiffre d'Affaires potentiel qu'un client peut apporter à une entreprise. Le marketing " one to one " engagera ses efforts en direction des clients dont la valeur stratégique est la plus forte.
Ces 5 points clés permettent de déterminer les caractéristiques principales du marketing "one to one " représentés dans le tableau suivant (EBG-1999)4.

\begin{tabular}{|c|c|}
\hline IDENTIFICATION & $\begin{array}{l}\text { Inventorier l'ensemble des informations disponibles dans les différentes bases } \\
\text { de données. } \\
\text { Développer des moyens de collectes de nouvelles données sur le profil et les } \\
\text { attentes du client : } \\
\text { - Demande d'information. } \\
\text { - Traitement des réclamations. } \\
\text { - Service après-vente. } \\
\text { - Achat simple ou répété. }\end{array}$ \\
\hline DIFFÉRENCIATION & $\begin{array}{l}\text { Adapter son offre en la différenciant à partir des besoins exprimés par les } \\
\text { consommateurs : } \\
\text { - Prise en compte des besoins particuliers de chaque client. } \\
\text { - Prise en compte de la valeur distincte de chaque client. }\end{array}$ \\
\hline INTERACTION & $\begin{array}{l}\text { Construire une relation avec le client, tout en estimant la valeur du client pour } \\
\text { elle-même : } \\
\text { - Prise en compte des informations-clients recueillies. } \\
\text { - Coordination des activités de l'entreprise pour considérer chaque client } \\
\text { individuellement. }\end{array}$ \\
\hline $\begin{array}{l}\text { PERSONNALISATION } \\
\text { DE L'OFFRE }\end{array}$ & $\begin{array}{l}\text { Personnaliser l'approche et les produits présentés en offrant un avantage } \\
\text { basé sur les besoins réels du client. }\end{array}$ \\
\hline
\end{tabular}

Cette orientation marketing à forcément des incidences importantes sur la communication commerciales de l'entreprise.

L'intégration du Marketing et de la Communication "one to one "dans la stratégie de développement de l'entreprise permettent d'avoir un consommateur individualisé et mieux connu ; demandeur d'un message personnalisé. Avec la croissance des TIC au sein de l'entreprise les zones de chalandises ne sont plus géographiques, mais typologiques. En effet, l'interactivité offre la possibilité de connaître préci- sément chaque individu, de collecter un nombre considérable d'informations, de les traiter et de formaliser un maximum de réponses adaptées à des cibles démultipliées.

C'est à partir de la collecte et de la gestion de ces mégabases de données en direct et en continu que les annonceurs seront amenés à définir une offre produit et communication en fonction du récepteur. Au lieu de trouver des consommateurs pour ses produits, l'entreprise devra véritablement trouver des produits pour des consommateurs pluriels. La communication com- 
merciale de l'entreprise doit de ce fait évoluer vers un véritable dialogue direct avec le consommateur, avec comme seuls messages acceptés ceux qui le concernent personnellement en fonction de ses attentes, ses devoirs et de ses référents socioculturels.

L'interactivité, avec la création de mégabases de données, permet de généraliser et d'amplifier cette démarche, d'en faire un véritable outil de conception, de commercialisation de produits et de création d'une relation personnalisée. La marque peut aussi prendre en compte, pour établir son discours, non seulement les attentes vis-à-vis des produits, mais aussi les aspirations émotionnelles et culturelles des individus, c'est-à-dire créer une véritable relation de marque personne à personne humaine. L'interactivité permet au consommateur de retrouver une certaine richesse relationnelle et réactive, telle qu'elle existait dans l'acte de vente individuelle chronique et de devenir un véritable " consommateur". De ce fait, même si celui à la tendance à " zapper " avec les différents supports médiatiques, il sera aussi prêt à " libérer " du temps au sein de l'espace relationnel d'une marque en fonction de la richesse, la pertinence et l'intérêt par rapport à ses attentes du message commercial.

La communication interactive permet de développer un véritable marketing relationnel de fidélisation dans lequel la dimension réactive est très importante. L'entreprise à travers ses marques soit ici démontrer qu'elle peut en permanence proposer des améliorations répondant immédiatement aux attentes des clients et créer en permanence de la valeur pour ce dernier.

\subsection{Les actions \\ promo-relationnelles : \\ facteur de convergence \\ entre les stratégies \\ promotionnelles et de \\ fidélisation de la clientèle \\ développée par les marques \\ fabricants.}

La communication interactive permet par l'intermédiaire d'outils comme le téléphone portable de développer des actions promo-relationnelles. Celles-ci vont renforcer l'impact des NIP ainsi que des promotions à contenu d'image ou niveau de la consolidation du capital marque des fabricants. Elles permettent également de concilier les notions de promotion et de fidélisation en direction de la clientèle.

Selon une enquête menée par l'institut Forrester Research à la fin de l'année 2001 auprès de 205 directeurs marketing, $64 \%$ déclaraient n'avoir jamais utilisé le SMS marketing et seuls $9 \%$ avaient eu recours à cette technique. La tendance s'inverse aujourd'hui puisque $56 \%$ avaient l'intention de mener des campagnes de marketing mobile à fin 2003. Côté utilisateurs, on dénombre aujourd'hui 42 millions de Français !

Le mobile offre en effet quatre atouts importants. L'affectivité, spécialement pour les jeunes, la continuité, la possibilité d'être joint quel que soit l'endroit et le moment ainsi que l'interactivité.

Il peut intégrer des actions qui vont de la publicité au marketing relationnel en passant par la promotion, les services clients. Il permet une relation durable, permanente et en tout lieu en rapprochant la marque du consommateur et en augmentent les opportunités de dialogue. Le mobile permet de 
N.I.P. (Nouveaux instruments promotionnels)

et promotions des marques : vers une synergie nécessaire

véhiculer des messages personnalisés et de "mettre une marque dans toutes les poches " à un coût économique faible surtout chez les moins de 25 ans qui représentent la moitié des émetteurs de SMS.

L'entreprise pourra développer une stratégie de SMS " push an pull " permettant à la fois d'animer, de créer du trafic, d'augmenter les ventes ; mais aussi de fidéliser et de récompenser dans une optique de Marketing Relationnel. Nous pouvons citer le cas de l'entreprise Nestlé qui a confié à Mobile et Permission filiale d'Orange une action promo-relationnelle à travers un jeu gratuit destiné au 13-25 ans avec les céréales Crunch. Conçu autour de l'univers du film "Le Seigneur des Anneaux ", le jeu se déroule en deux temps : un instant gagnant par SMS avec rebond vers un call center et une participation par envoi postal. Cette opération permet à Nestlé Crunch de répondre à un double objectif : animer le produit en linéaire grâce à un packaging spécifique et créer de la visibilité auprès des adolescents. Le client peut s'inscrire dans le club Crunch. Contrepartie de l'inscription : un don immédiat (gratuité produit, un service additionnel, place de cinéma, etc...) car le consommateur ne fait plus rien gratuitement.

Grâce au mobile la promotion n'est plus seulement un acte en vue de créer du trafic et d'augmenter le volume, elle devient potentiellement un acte de recrutement et de fidélisation. La promesse d'immédiateté séduit la cible des jeunes. En outre, le nombre de joueurs est en moyenne cinq fois plus élevé qu'avec la méthode papier traditionnelle. Plus de $10 \%$ des joueurs se sont inscrits au club Crunch soit un taux de retour moyen très élevé. Avec la mise en place du "Multimédia Messaging Service" (MMS) associant à l'écrit, l'image et le son, les actions promorelationnelle devraient se développer fortement.

\section{Une esquisse \\ de rapprochement \\ des concepts de promotion \\ et de fidélisation au travers \\ des NIP et de la promotion \\ à contenu d'image}

\subsection{La nouvelle politique promotionnelle des distributeurs}

Les fabricants ont tendance à considérer les enseignes comme peu légitimes dans le développement de stratégies promotionnelles, celles-ci ayant longtemps été l'apanage des marques.

Toutefois, ce sont pourtant les distributeurs qui ont, les premiers mis en place et développés les techniques d'animation commerciale et de promotion des ventes (premières opérations se ventes à primes en 1898 par Casino et système de points proposés par Coop). Il s'agissait déjà de communication persuasive permettant de mieux maîtriser la relation commerciale avec le consommateur.

L'intégration du libre-service et surtout la création du concept d'hypermarché par l'enseigne Carrefour en 1963 change la donne. La distribution abandonne son rôle de "commerce" pour créer des usines à vendre 
(A. Parmentelot 2004)5. Dans cet environnement neutre et indifférencié, les marques saisissent l'opportunité de développer auprès des consommateurs des avantages différentiels en exploitant leurs packagings, la PLV et aussi la concentration des ventes dans un nombre de lieux limité.

À partir des années 1990, les distributeurs s'engagent de plus en plus dans des actions de différenciation concurrentielle et de fidélisation de la clientèle. Ils développent dans des outils promotionnels (Tête de Gondole - PLV), des actions de marketing direct en collaboration avec les fabricants ou d'autres médias alternatifs comme le marketing situationnel (F. DEBOS 1993)6 et expérentiel du point de vente (O. BADOT 2003) 7 (B.DAUCE-S.RIEUNIER) ${ }^{8}$.
De grandes enseignes et marques fabricants développent conjointement des actions promotionnelles et ce de façon structurelle.

À l'heure actuelle nous pouvons constater la coexistence des "VIP " (Vieux Instruments Promotionnels) qui sont des techniques hard-selling proposées par les marques (lots, réductions, gratuité, etc...), avec répercussion par les distributeurs des avantages financiers et communication de mises en avant et prospectus, communication financée par la coopération commerciale avec les NIP.

Le principe des NIP ne repose pas sur de grandes nouveautés techniques mais sur le cumul et l'obtention différée des avantages. Les techniques entrant dans le champ des NIP sont présentées dans le tableau suivant.

5. A. PARMENTELOT 2004 "La mercatique stratégique des réseaux " - Édition Fontaine PICARD - Collection CUBIX 382 pages

6. F. DEBOS juin 1993 "L'impact des facteurs situationnels sur la fréquence et les moments d'achat des produits courants le cas de la grande distribution "Thème de Doctorat 377 pages.

7. O. BADOT 2003 "Le marketing expérentiel du distributeur " pp 284-297. Ouvrage collectif "Le marketeur " PEARSON EDUCATION 456 pages.

8. B. DAUCE - O.RIEUNIER 2002 "Le marketing sensoriel du point de vente " Recherche et application en marketing " Vol $17-\mathrm{N}^{\circ} 4-$ pp $45-65$. 
N.I.P. (Nouveaux instruments promotionnels) et promotions des marques : vers une synergie nécessaire

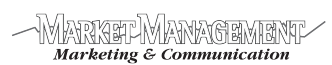

\begin{tabular}{|c|c|c|c|c|}
\hline $\begin{array}{c}\text { Cartes } \\
\text { fidélisation }\end{array}$ & $\begin{array}{c}\text { Bon d'achat } \\
\text { ticket }\end{array}$ & $\begin{array}{c}\text { Carte } \\
\text { Cagnotte }\end{array}$ & Lot virtuel & $\begin{array}{c}\text { Offre } \\
\text { fédératives }\end{array}$ \\
\hline $\begin{array}{l}\text { Points obtenus sur } \\
\text { les montants } \\
d^{\prime} \text { 'achat et sur cer- } \\
\text { tains produits. }\end{array}$ & $\begin{array}{l}\text { Bon d'achat obtenu } \\
\text { sur l'achat de cer- } \\
\text { tains produits (par- } \\
\text { fois rayon). A valoir } \\
\text { lors de prochains } \\
\text { achats. }\end{array}$ & $\begin{array}{l}\text { Cumul des bons } \\
d^{\prime} \text { achat sur une } \\
\text { carte collecteur. } \\
\text { Cumulable dans le } \\
\text { temps. }\end{array}$ & $\begin{array}{l}\text { Réduction automa- } \\
\text { tique en caisse sur } \\
\text { une combinaison } \\
\text { produits. }\end{array}$ & $\begin{array}{l}\text { Offres fédérant des } \\
\text { marques dans un } \\
\text { même système. } \\
\text { Développées par les } \\
\text { enseignes. }\end{array}$ \\
\hline $\begin{array}{l}\text { Transformables en } \\
\text { cadeaux ou bons } \\
\mathrm{d}^{\prime} \text { achat (valeur } \\
0.5 \% \text { des achats). }\end{array}$ & $\begin{array}{l}\text { Valeur d'environ } \\
15 \% \text { du produit } 20 \\
\text { ou } 25 \% \text { lors d'opé- } \\
\text { rations spéciales. }\end{array}$ & $\begin{array}{l}\text { Valeur de } 15 \text { ou } \\
20 \% \text {. Bonus de } \\
25 \% \text { sur carte } \\
\text { Leclerc. }\end{array}$ & $\begin{array}{l}\text { Valeur } 15 \text { à } 20 \% \text {. } \\
\text { De plus en plus } \\
25 \% \text { (2e à moitié } \\
\text { prix), ou } 33 \% \text { ( } 3 \\
\text { pour } 2 \text { ). }\end{array}$ & $\begin{array}{l}\text { ODR* } 100 \% \text { rem- } \\
\text { boursés (10 à } 50 \\
\text { produits). Colis } \\
\text { d'échantillon (10 à } \\
50 \text { ). } \\
\text { * Offre de remboursement }\end{array}$ \\
\hline $\begin{array}{l}\text { U, Champion, Atac, } \\
\text { Casino, Match, } \\
\text { Shopi, Géant, Cora } \\
\text { (sur carte de paie- } \\
\text { ment). }\end{array}$ & $\begin{array}{l}\text { Ticket Leclerc, Ticket } \\
\text { Cash Carrefour } \\
\text { (arrêt en } \\
\text { mars 2004). } \\
\text { Ticket + Champion } \\
\text { et Bon U (système } \\
\text { écobon). }\end{array}$ & $\begin{array}{l}\text { Leclerc, Auchan } \\
\text { (Waaoh), nouvelle } \\
\text { carte de fidélité } \\
\text { Carrefour, } \\
\text { Intermarché. }\end{array}$ & $\begin{array}{l}\text { Carrefour (en bais- } \\
\text { se), Géant, Auchan, } \\
\text { Cora. }\end{array}$ & $\begin{array}{l}\text { ODR (Intermarché, } \\
\text { Atac Carrefour, } \\
\text { Champion). } \\
\text { Echantillons : colis } \\
\text { bébé, RV gagnants } \\
\text { Intermarché. } \\
\text { BR* (en baisse) } \\
\text { * bon de réduction. }\end{array}$ \\
\hline
\end{tabular}

Nous pouvons à titre d'exemple citer le cas du nouveau programme de fidélité lancé par Carrefour en pour répondre aux "tickets leclerc " qui ont eu un impact très fort auprès des clients. Le client sélectionne 25 produits dans une liste de 200. Chaque achat de ces produits génère une "réduction libre " créditée sur un compte et chaque mois sous certaines conditions, un chèque de fidélité est adressé à ce client. Ce dernier bénéficie en parallèle de bons de réduction, de réductions exclusives et de $10 \%$ de réduction supplémentaire en cas de paiement avec la carte de l'enseigne, la carte Pass. Même s'il peut paraître complexe, ce programme, résume en ces quelques lignes, comporte un double avantage. En premier lieu, il ôte à la promotion son caractère habituellement très ponctuel, en l'institutionnalisant sur une année. Ce qui peut permettre de susciter des " habitudes " fidélisatrices (J.M LEHU - 2003)9. Notamment puisqu'en second lieu, il incite le client à se "déclarer " de fait pendant un an, fidèle à 25 produits de marque. Mais, il requiert une imposante logistique de l'information et de la gestion des chèques et autres bons. De plus le risque est de voir les titulaires de cette carte de fidélité effectuera seulement chez Carrefour les achats pour lesquels il bénéficiera de réductions promotionnelles. Il ne s'agira pas d'une réelle fidélisation de la clientèle par le biais de ces outils promotionnels. Même si les NIP ne sont pas utilisés de façon optimale, par les distributeurs (coûts

9. J.-M LEHU 2003 "Stratégies de fidélisation " Éditions d'Organisation. 
importants, complexification de l'offre pour le client avec risque de publicité trompeuse, financement par les marques) ; ils constituent une première tentative d'associations des techniques promotionnelles avec des outils de fidélisation du consommateur. Leur mise en place doit être plus fondée sur une véritable réflexion marketing associant Enseignes et Fabricants tout en soulignant leurs spécificités et ne pas être seulement des outils de développement de leur CA à court terme.

\subsection{Le développement \\ de la promotion à contenu d'image par les marques fabricant}

Les actions promotionnelles sont souvent considérées comme des outils inadaptés en terme de gestion de l'image d'un produit; notamment en comparaison avec la publicité-média. Il pourrait même exister un risque d'affaiblissement et de dilution du capital de marque lors d'une utilisation trop intensive des promotions.

Or certaines formes de communication promotionnelle peuvent être utilisées pour promouvoir l'image de marque en intégrant en terme de contenu et d'accroche l'équivalent d'une annonce publicitaire efficace. À ce niveau nous pouvons citer le cas de Kellogg's, qui utilise le dos des boîtes de céréales un peu comme une quatrième de couverture, qui sera vue par le consommateur pendant le petit déjeuner (cette tactique est d'ailleurs reprise par des marques distributeurs comme "brin du jour" de Leclerc). L'entreprise utilise ici deux facteurs de cohérence :
- Une cohérence avec l'image que l'ont cherche à donner du produit. Par exemple Frosties vent développer une image jeune et dynamique à travers le sport donc va proposer de leçons de sports de glisse (ski, surf, skate). Pour Special K, les promotions sont centrées sur la beauté, la sveltesse, la santé, la ligne. Il s'agira alors de présenter des cures de thalassothérapie ou des opérations de co-branding avec weightwatchers.

- Une cohérence dans le temps avec les mêmes objectifs, les mêmes thèmes qui sont déclinés de façon méthodique et rigoureuse permettant une action de renforcement de l'image de marque sans être vécu comme du "matraquage publicitaire".

Ces deux facteurs sont renforcés par des messages très cohérents avec les promotions habituelles lorsque l'entreprise n'est pas en situation d'action promotionnelle; et ce toute l'année (messages diététiques, indication d'exercices physiques, etc...). Ces " non-promotions " soulignent, de façon paradoxale, deux composantes essentielles des promotions Kellogg's : l'accompagnement d'image donné systématiquement, avec une grande qualité d'exécution, à chaque promotion, en s'appuyant, encore une fois, sur le riche support que constitue une image de grand format $(19 \mathrm{~cm}$ sur $26 \mathrm{~cm}$ ) vue chaque matin, pendant plusieurs minutes, sur la table du petit déjeuner ; l'habitude donnée au consommateur de lire activement cette boîte, plutôt que de la voir passivement, en background. Deux autres techniques promotionnelles identifiées par Gilles 
N.I.P. (Nouveaux instruments promotionnels) et promotions des marques : vers une synergie nécessaire

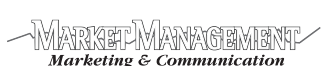

Marketing E communication
LAURENT10 peuvent être prises en compte. Il s'agit de "l'agglutination " et de la "sidération ". Dans le premier cas il s'agit d'offrir un objet promotionnel (prime, cadeau, prix de loteries etc...) à forte valeur d'image permettant de valoriser le capital marque du produit ou service considéré. La sidération correspond à des opérations très décalées des actions promotionnelles habituelles comme l'offre d'un petit canard en plastique aux clients des hôtels de la chaîne Ramada (Alan TOOP - 1992)11.

Comme nous le voyons le contenu de ces promotions est riche sur le plan communicationnel. Elles peuvent être un vecteur de renforcement de l'image de marque auprès des cibles virées par l'entreprise et contribuent à la fidélisation de sa clientèle.

\section{Conclusion}

Nous pouvons constater que depuis ces dernières années le nombre d'actions promotionnelles n'a cessé d'augmenter. Cette situation est à rapprocher d'un certain ralentissement économique qui implique pour les fabricants et les distributeurs le besoin de déclencher chez le consommateur des achats, notamment d'impulsion, voire de permettre au moins de générer du trafic sur le lieu de vente. A ce niveau les différents outils promotionnels étudiés s'inscrivent dans cette logique ou l'émulation concurrentielle induit une surenchère constante. Nous le voyons par l'intermédiaire des NIP qui (ré)inventent chaque jour de nouveaux avantages pour séduire un chaland hésitant et sollicite de toutes parts. Toutefois, pendant cette même période d'augmentation des promotions en tout genre, on n'a jamais tant parlé de fidélisation. Jamais déployé autant de systèmes informatiques censés assurer un Customer Relationship Management des plus performants. Il ne faut pas oublier en effet qu'un client fidèle revient à priori cinq à sept fois moins cher qu'un client non fidèle. Cette apparente contradiction peut s'expliquer par le fait que globalement les marques ont bien sur, besoin de fidéliser leurs clients mais aussi de façon plus globale de leviers promotionnels pour gérer leurs développements, notamment dans le cadre d'objectifs stratégiques et relationnels. Sans ces leviers, il n'y a pas de stratégie marketing opérante. Les enseignes de distribution doivent aussi maintenir la position des marques fabricants (même si elles peuvent concurrencer leurs marques) par rapport aux attentes des consommateurs mais aussi en tant qu'élément différenciateurs du Hard Discount. Tout en utilisant des médias non contrôlés par les enseignes ; massmédias, mais surtout médias directs : marketing direct (avec clubs), boîtes aux lettres, marketing situationnel, marketing viral, internet ; les marques fabricants doivent développer en collaboration avec les distributeurs des actions de communication persuasive de type promotionnel.

Nous pouvons citer Nestlé avec ses opérations chocolat, chez Carrefour ou Auchan, le Comité chandeleur avec ses animations-dégustations de 
crêpes dans les principaux hypermarchés ou encore Dim avec les " beautiful days ", véritable boutique temporaire avec animation, chéquier de bon de réduction et primes. Comme nous le voyons ; dans le cas des marques fabricants de produits de grande consommation, les actions de promotion et de fidélisation ne sont pas objectivement opposables, d'où leur possible coexistence. Mais elles peuvent en revanche aisément se contrarier et même se nuire, au grand détriment de la marque. Mal organisées, des actions promotionnelles peuvent très vite remettre en question la fidélité naissante d'un consommateur envers la marque, voire altérer le positionnement et l'image de cette dernière dans les cas les plus extrêmes. Mal orchestrée, une stratégie de fidélisation peut devenir à ce point pesante, qu'elle nuira à l'efficacité d'une opération promotionnelle. Il faut alors créer une véritable synergie entre ces deux catégories d'actions commerciales. Les actions promotionnelles doivent s'intégrer au sein de la stratégie de gestion de la marque. En parallèle les stratégies de fidélisation de la clientèle développées par les marques vont intégrer des actions promotionnelles comme les promotions à contenu d'images, les NIP ou les actions promo-relationnelles. Promotion et fidélisation ne doivent pas être élaborées de façon distinctes (par la direction commerciale pour la première et par la direction marketing pour la seconde) mais doivent être pensées ensembles autour de la marque qui servira de fil conducteur tout le long de la chaîne de valeur au sens de Porter ${ }^{12}$. Les entreprises pourront ainsi contribuer à la croissance et à la protection du capital de la marque.

\section{Références bibliographiques}

Badot O. (2003), "Le Marketing Expérientiel du distributeur » pp. 284-297, In ouvrage collectif, «Le Marketeur », Pearson Éducation, 456 pages.

Dauce B., Rieunier O. (2002), "Le Marketing sensoriel du point de vente ", Recherche et Application en Marketing, Volume $17 \mathrm{n}^{\circ}$ 4, pp. 45-65.

Debos F. (Sept. 2004), "L'impact de l'interactivité sur la communication commerciale de l'entreprise : vers l'animation de clans d'audience", 14 e Conférence Internationale du RESER, Castres, Volume 1, pp. 539-560, 580 pages.

Debos F. (juin 1993), " l'impact des facteurs situationnels sur la fréquence et les moments d'achat des produits courants : le cas de la grande distribution ", Thèse de Doctorat, 377 pages.

EBG (1999) "le guide du commerce électronique " PUBLI UNION. Cité dans l'article de Loïc ROCHE (2001) «Internet 2001, Défis d'hier et à venir- Etat des lieux », Revue Humanisme et Entreprise ${ }^{\circ} 248$ pp. 77-100.

Laurent G (1999), "La promotion: un outil pour manager l'image de marque". La Revue des Marques - n०25 - pp. 33-35.

Lehu J.-M. (2003), «Stratégies de Fidélisation », Édition d'Organisation.

Leutheusser L. (1988), "Defining, Measuring and Managing Brand Equity ", Working Paper, pp. 88, 104 - Marketing Science Institute.

Parmentelot A. (2004), « La Mercatique stratégique des Réseaux », Édition Fontaine Picard, Collection Cubix, 382 pages.

Peppers D., Rogers M (1999), "Le One to One : valorisez votre capital client », Édition d'Organisation, 312 pages.

Porter M. (1997), « L'avantage Concurrentiel », Édition Dunod.

Toop A. (1992), "European Sales Promotions Great Campaigns in Action » Édition Kogan Page.

12. M. PORTER Sept 1997 - "L'avantage concurrentiel " - Édition DUNOD. 